\title{
Capsule Commentary on Landon et al., "Trends in Diabetes Treatment and Monitoring Among Medicare Beneficiaries"
}

\author{
Seth A. Berkowitz, MD MPH \\ Division of General Medicine and Clinical Epidemiology, Department of Medicine, and the Cecil G. Sheps Center for Health Services Research, \\ University of North Carolina School of Medicine, Chapel Hill, NC, USA.
}

$\mathrm{J}$ Gen Intern Med 33(4):498

DOI: $10.1007 / \mathrm{s} 11606-018-4339-4$

(c) Society of General Internal Medicine 2018

I n this study of Medicare claims (2007-2014) from older 1 individuals with type 2 diabetes, Landon et al. ${ }^{1}$ highlight a mix of encouraging and discouraging findings. One caveat in interpreting their findings is that diabetes treatment guidelines are consensus-based, ${ }^{2,3}$ and we often do not know what proportion of patients the guidelines apply to, or what proportion of patients receiving particular treatments is appropriate.

Encouraging findings in the study are the increased use of metformin as initial pharmacotherapy (from 50 to $70 \%$ ), the increased use of stains, and the decreased use of long-acting sulfonylureas. Discouraging findings include the potential overuse of blood glucose self-monitoring and safety concerns related to the complexity of insulin regimens in older and sicker individuals. Given the presumption that other regimens might be safer and equally effective, insulin treatment in older adults deserves particular attention in future work.

An area of uncertainty for diabetes treatment is selecting medications to add to metformin. The ongoing GRADE randomized clinical trial ${ }^{4}$ seeks to inform this decision, but until results are available, clinicians will have to rely on choosing agents based on side effects, cost, and patient preference, as most agents offer similar reductions in glycemia. An emerging consideration however is cardiovascular risk reduction, particularly seen with GLP-1 and SGLT-2 agents. ${ }^{5}$ Whether this evidence is generalizable to individuals at lower risk than those included in the trials (a majority of those with diabetes), and whether the high cost of these agents is justified, remains to be seen.

Median out of pocket medication costs nearly halved during the study period, to $\$ 105 /$ year. However, mean costs increased during the study period, driven by the use of expensive medications in a small proportion of individuals. While this study cannot determine whether that spending is justified, this is another key area for further research.

For those studying trends in diabetes treatment, particularly for older adults, this study offers an important snapshot of the changing landscape. Further, it highlights questions about complicated and expensive treatment regimens as key areas for future study, as we make concerted efforts to offer safer and more effective diabetes care.

Corresponding Author: Seth A. Berkowitz, MD MPH; Division of General Medicine and Clinical Epidemiology, Department of Medicine, and the Cecil G. Sheps Center for Health Services Research University of North Carolina School of Medicine, Chapel Hill, NC, USA (e-mail: Seth_Berkowitz@med.unc.edu).

\section{Compliance with Ethical Standards:}

Conflict of Interest: I declare that I have no conflicts of interest to disclose.

\section{REFERENCES}

1. Landon B, Zaslavsky A, Souza J, Ayanian J. Trends in diabetes treatment and monitoring among Medicare beneficiaries. J Gen Intern Med. https://doi.org/10.1007/s11606-018-4310-4.

2. American Diabetes Association. 8. Pharmacologic approaches to glycemic treatment: standards of medical care in diabetes-2018. Diabetes Care.. 2018;41(Suppl 1):S73-S85. https://doi.org/10.2337/dc18-S008.

3. Guidelines abstracted from the American Geriatrics Society Guidelines for improving the care of older adults with diabetes mellitus: 2013 update. J Am Geriatr Soc. 2013;61(11):2020-2026. https://doi.org/10.1111/jgs. 12514.

4. Nathan DM, Buse JB, Kahn SE, et al. Rationale and design of the glycemia reduction approaches in diabetes: a comparative effectiveness study (GRADE). Diabetes Care. 2013;36(8):2254-2261. https://doi.org/ 10.2337/dc13-0356.

5. Arnold SV, Inzucchi SE, Tang F, et al. Real-world use and modeled impact of glucose-lowering therapies evaluated in recent cardiovascular outcomes trials: an NCDR® research to practice project. Eur J Prev Cardiol. 2017;24(15):1637-1645. https://doi.org/10.1177/ 2047487317729252. 